\title{
Avaliação das reações adversas e do desfecho clínico em pacientes tratados para tuberculose multirresistente em um hospital de referência na cidade de Belém, Pará
}

\author{
Evaluation of adverse reactions and clinical outcome in patients treated for multidrug- \\ resistant tuberculosis at a referral hospital in the city of Belém, Pará
}

Evaluación de las reacciones adversas y del desenlace clínico en pacientes tratados para tuberculosis multirresistente en un hospital de referencia en la ciudad de Belém, Pará

Dayanne Aline Bezerra de Sá1, Christe Ellen Batista Fanzlau¹, Maitê dos Santos Feitosa1*, Maisa dos Santos Feitosa', Renan Reis Caldas ${ }^{1}$, Nayara Henestyne Cantuária Figueiredo ${ }^{1}$, Victor Hugo Monteiro Pontes ${ }^{1}$, Carlos Augusto Abreu Alberio².

\section{RESUMO}

Objetivo: Avaliar o impacto de reações adversas no desfecho clínico em pacientes tratados para tuberculose multirresistente. Metodologia: Estudo observacional analítico do tipo coorte retrospectivo dos casos de TBMR cadastrados no SITE-TB, que realizaram tratamento no período de janeiro de 2010 a dezembro de 2015 . Resultados: Foram avaliados 152 pacientes com uma média de idade de 40,2 anos, predominando o sexo masculino $(65,1 \%)$. A maioria dos pacientes se concentra na região metropolitana de Belém $(73,7 \%)$, com predomínio de desempregados (25\%). Parcela significativa apresentou resistência secundária (88,2\%). Em relação ao consumo de drogas, o uso do álcool foi mais frequente $(20,4 \%)$, seguido por drogas ilícitas (16,5\%) e tabaco (5,3\%). A presença de comorbidades foi de $27,6 \%$, sendo que o Diabetes Mellitus foi a mais comum (23\%). Metade dos pacientes do estudo apresentou reações adversas e as mais frequentes foram artralgia $(31,6 \%)$, epigastralgia $(15,1 \%)$ e neuropatia periférica $(5,3 \%)$. O desfecho clinico favorável ocorreu em $77 \%$ dos casos. Dentre os pacientes que apresentavam comorbidade, a maioria apresentou desfecho favorável $(88,1 \%)$. Assim como, os que apresentaram efeitos adversos também tiveram mais desfecho favorável $(79,0 \%)$, entretanto, esse dado não obteve significância estatística $(p=0,563)$. Os usuários de drogas ilícitas e tabaco apresentaram maior frequência de desfecho desfavorável (56,0\% e 62,5\%, respectivamente). Conclusão: A tuberculose multirresistente se mantém como um importante problema de saúde pública, com frequência elevada, principalmente entre a população economicamente ativa.

Palavras-chave: Tuberculose multirresistente (TBMR), Reações adversas, Desfecho clínico.

\begin{abstract}
Objective: To evaluate the impact of adverse reactions on the clinical outcome in patients treated for multiresistant tuberculosis. Methodology: An observational, retrospective cohort study of cases of MRT registered on SITE-TB, which underwent treatment from January 2010 to December 2015. Results: A total of 152 patients who presented with a mean age of 40,2 years old, predominantly male $(65.1 \%)$. The majority of the patients was concentrated in the metropolitan region of Belém $(73.7 \%)$, with a predominance of the unemployed $(25 \%)$. Significant share presented secondary resistance $(88.2 \%)$. Regarding drug use, alcohol use was more frequent $(20.4 \%)$, followed by illicit drugs $(16.5 \%)$ and tobacco $(5.3 \%)$. The presence of comorbidities was $27.6 \%$, and Diabetes Mellitus was the most common (23\%). Half of the patients in the study
\end{abstract}

\footnotetext{
${ }^{1}$ Médico da unidade de pronto atendimento de Icoaraci. * E-mail: maytedossantos@hotmail.com

2 Professor adjunto da Faculdade de Medicina da UFPA.
} 
had adverse reactions and the most significant were arthralgia (31.6\%), epigastric pain (15.1\%) and peripheral neuropathy (5.3\%). The favorable clinical outcome was predominant in $77 \%$ of the cases. Among the patients presenting with comorbidity, the majority presented a favorable outcome (88.1\%). As well as those with adverse effects also had a more favorable outcome $(79.0 \%)$, however this data did not reach statistical significance $(p=0.563)$. The users of illicit drugs and tobacco showed a higher frequency of unfavorable outcomes $(56.0 \%$ and $62.5 \%$, respectively). Conclusion: Multidrug-resistant tuberculosis remains an important public health problem, with high frequency, especially among the economically active population.

Key words: Multidrug-resistant tuberculosis (MDR-TB), Adverse reactions, Clinical outcome.

\section{RESUMEN}

Objetivo: Evaluar el impacto de reacciones adversas en el desenlace clínico en pacientes tratados para tuberculosis multirresistente. Metodología: Estudio observacional analítico del tipo cohorte retrospectivo de los casos de TBMR en el SITE-TB, en el período de enero de 2010 a diciembre de 2015. Resultados: Se evaluaron 152 pacientes, con una media de edad de 40, 2 años, predominando el sexo masculino (65,1\%). La mayoría de los pacientes se concentra en la región metropolitana de Belém (73,7\%), con predominio de desempleados (25\%). La proporción significativa presentó resistencia secundaria $(88,2 \%)$. En cuanto al consumo de drogas el uso del alcohol fue más frecuente (20,4\%), seguido por drogas ilícitas (16,5\%) y tabaco $(5,3 \%)$. La presencia de comorbilidades fue baja $(27,6 \%)$, siendo que la Diabetes Mellitus fue la más común (23\%). La mitad de los pacientes del estudio presentó reacciones adversas y las más frecuentes fueron artralgia $(31,6 \%)$, epigastralgia $(15,1 \%)$ y neuropatía periférica $(5,3 \%)$. El resultado clínico favorable se produjo en el $77 \%$ de los casos. Entre los pacientes que presentaban comorbilidad, la mayoría presentó un resultado favorable $(88,1 \%)$. Así como, los que presentaron efectos adversos también tuvieron más desenlace favorable $(79,0 \%)$, sin embargo, ese dato no obtuvo significancia estadística $(p=0,563)$. Los usuarios de drogas ilícitas y tabaco presentaron una mayor frecuencia de desenlace desfavorable $(56,0 \%$ y $62,5 \%$, respectivamente). Conclusión: La tuberculosis multirresistente se mantiene como un importante problema de salud pública, con frecuencia elevada, principalmente entre la población económicamente activa.

Palabras clave: Tuberculosis multirresistente (TBMR), Reacciones adversas, Desventaja clínica.

\section{INTRODUÇÃO}

A Tuberculose (TB) permanece desde a antiguidade entre as doenças infectocontagiosas que mais acometem a humanidade. Depois de um século da identificação do bacilo Mycobacterium tuberculosis como o agente causador da doença e cerca de 50 anos após a descoberta de um tratamento medicamentoso específico e eficaz, a TB permanece como um grave problema de saúde pública de relevância mundial (BRASIL, 2007).

Embora a incidência global da doença esteja em lento declínio, ela ainda permanece como uma das principais causas de morbidade e mortalidade mundial. Em muitas regiões do planeta, incluindo partes da Europa, a alta incidência de TB é um forte indicador de baixos níveis socioeconômicos e de assistência à saúde deficiente (LANGE e MIGLIORI, 2012).

O Brasil é um dos 22 países priorizados pela OMS que concentram $80 \%$ da carga mundial de TB, ocupando 19a posição em relação ao número de casos (WHO, 2009). Aproximadamente 9,4 milhões de novos casos e 1,7 milhões de mortes atribuídos a TB são encontrados por ano ao redor do mundo (RUDEEANEKSIN et al., 2012).

A emergência dos casos de resistência está normalmente associada ao tratamento irregular. $O$ uso inadequado dos medicamentos, a baixa adesão ao tratamento, o abandono, o fornecimento irregular dos fármacos e a prescrição inadequada levam a concentrações abaixo dos níveis terapêuticos recomendados, 
propiciando a multiplicação dos bacilos fármacos-resistentes preexistentes (resistência adquirida). A infecção por estes bacilos resistentes nas pessoas que nunca tiveram a doença é chamada de tuberculose resistente primária (WHO, 2008; BRASIL, 2011).

Dentre as formas de resistência, a tuberculose multirresistente (TB-MDR), caracterizada pela resistência aos principais fármacos de primeira linha utilizados no tratamento, a rifampicina e a isoniazida, constitui um grave problema de saúde pública. A TB-MDR apresenta várias limitações relacionados ao seu tratamento: baixa eficácia, tempo muito prolongado de tratamento, reações adversas frequentes e fontes limitadas de drogas com custo extremamente elevados dos medicamentos de segunda linha (MIGLIORI et al., 2008).

Em 2008, estima-se que 390.000 a 510.000 novos casos de TB-MDR surgiram no mundo (WHO, 2011). No Brasil, de 2000 a 2007, 2.690 casos de TB-MDR foram registrados, com taxa de mortalidade de aproximadamente $8,6 \%$ (ORENSTEIN et al., 2009). A maioria dos casos de multirresistência no mundo e no Brasil é adquirida por tratamentos irregulares e abandono. Para o tratamento desses casos, é necessário 0 uso de medicamentos injetáveis e de fármacos orais de segunda linha, com efetividade de cerca $60 \%$ de cura (BRASIL, 2007; BRASIL, 2011).

No Brasil, o esquema de tratamento atualmente recomendado para os casos de TB-MDR é padronizado e composto por cinco fármacos: na primeira fase ou fase intensiva, com duração de 6 meses, são utilizados a Estreptomicina, Levofloxacina, Etambutol, Pirazinamida e Terizidona, enquanto que na segunda fase ou fase de manutenção, com duração de pelo menos 12 meses, são utilizados somente a Levofloxacina, Terizidona e Etambutol (BRASIL, 2009).

A baixa adesão ao tratamento da TB, por sua vez, além de estar relacionada a aspectos socioeconômicos e culturais, tem nas reações adversas um de seus principais responsáveis. A ocorrência de reações adversas tem como principais causas: associação de vários fármacos no tratamento, interação com outros medicamentos, faixa etária do paciente, baixo peso, etilismo, coinfecção com HIV e a presença de doenças pré-existentes como insuficiências renal e hepática (WHO, 2008).

O monitoramento rigoroso dos pacientes em tratamento para TB-MDR é extremamente importante para que se possam identificar os pacientes com alto risco para a apresentação de reações adversas e garantir a intervenção precoce, o manejo adequado e o desfecho clínico favorável. Alguns dos aspectos importantes da monitorização clínica, laboratorial e funcional são: teste de gravidez antes de iniciar o tratamento, controle das funções renal e hepática, monitoramento eletrolítico, alteração da função tireoidiana, comprometimento da visão e audição. Este trabalho tem como objetivo avaliar o impacto das reações adversas ao tratamento da Tuberculose Multirresistente no desfecho clínico destes pacientes. (WHO, 2013; NATHANSON et al., 2005; TAG EL DIN et al., 2015).

\section{MÉTODOS}

Trata-se de um estudo observacional analítico do tipo coorte retrospectivo, realizado no ambulatório de tuberculose multirresistente em um Hospital Universitário em Belém-PA. Foram analisados neste estudo pacientes com o diagnóstico de tuberculose multirresistente, de ambos os gêneros, com idade igual ou maior que 18 anos, que realizaram o tratamento padrão para TBMDR e que aceitaram participar do estudo mediante assinatura do Termo de Consentimento Livre e Esclarecido. O estudo foi aprovado pelo Comitê de Ética em Pesquisa do Hospital Universitário João de Barros Barreto, ligado à Universidade Federal do Pará (CAAE no 62189916.7.0000.0017 - Parecer 1.988.872).

O diagnóstico de tuberculose multirresistente consiste em cultura positiva para espécies de micobactérias e teste de sensibilidade nos materiais biológicos dos indivíduos suspeitos de apresentar resistência aos medicamentos utilizados nos esquemas padronizados. A OMS define que são portadores de TB-MDR os indivíduos que apresentam doença causada por cepas com resistência in vitro à rifampicina e isoniazida. $O$ tratamento padrão para TB-MDR é composto pela associação de estreptomicina, etambutol, pirazinamida, levofloxacina e terizidona. 
O tamanho amostral foi composto por todos os pacientes que realizaram tratamento para tuberculose multirresistente de janeiro de 2010 a dezembro de 2015, com início e término do tratamento nesse período. Não foram considerados neste estudo indivíduos soropositivos para HIV, portadores de insuficiência renal, insuficiência hepática, doenças autoimunes, neoplasias e os pacientes que apresentaram mudanças no esquema terapêutico durante o período de tratamento.

As variáveis estudadas nesta pesquisa foram: variáveis demográficas (idade, gênero, cor, residência, escolaridade, profissão), variáveis clínicas (tipo de tuberculose multirresistente; história de tabagismo; alcoolismo e uso de outras drogas de abuso; comorbidades; efeitos adversos e desfecho de tratamento).

A tuberculose multirresistente é dividida em primária e secundária, resistência primária verifica-se em pacientes nunca tratados para TB, contaminados por bacilos previamente resistentes e a secundária verificase em pacientes com tuberculose inicialmente sensível, que se tornam resistentes após a exposição aos medicamentos (BRASIL, 2007). Os efeitos adversos pesquisados foram: náuseas e vômitos; epigastralgia; anafilaxia; convulsão; sintomas psicóticos; cefaleia; zumbido; insônia; neurite óptica; ototoxicidade; alterações dermatológicas; neuropatia periférica; artralgia.

Foram considerados os seguintes desfechos: desfecho favorável quando há alta do tratamento por cura, e desfecho desfavorável que abrange: alta por falência de tratamento, alta por abandono, alta por óbito.

As informações do trabalho foram retiradas do SITETB (Sistema de Informação de Tratamentos Especiais da Tuberculose), que é um banco de dados do Ministério da Saúde para a notificação e o acompanhamento dos casos de TB que têm indicação de tratamentos especiais, e dos prontuários dos pacientes atendidos no hospital universitário. Foi utilizada uma ficha clínica padronizada para cada paciente do estudo onde foram realizados os registros de todas as informações do protocolo de pesquisa.

Posteriormente, estas informações foram armazenadas em um banco de dados para a realização das análises estatísticas. Os dados foram processados no programa Microsoft Excel 2016. As variáveis qualitativas foram descritas segundos suas frequências absolutas e relativas. As variáveis quantitativas que apresentarem distribuição normal foram descritas por média e desvio padrão. Já as variáveis quantitativas que não apresentarem distribuição normal foram descritas por mediana e percentil $25 \%-75 \%$. O teste para avaliação da adequação à normalidade foi o de Shapiro-Wilk. Foi adotado significância estatística para valores de $p<=0,05$. O programa estatístico utilizado foi o Stata 12.0 .

\section{RESULTADOS}

Foram avaliados 152 indivíduos atendidos no ambulatório de Tuberculose multirresistente, de um hospital de referência na cidade de Belém, Pará, no período de janeiro de 2010 a dezembro de 2015. A média de idade foi de 40.2 anos $(\mathrm{dp}=12.5)$.

As características sócio epidemiológicas evidenciam que a maioria dos pacientes com TBMDR foram do sexo masculino $(65,1 \%)$, sendo que a região metropolitana de Belém - o maior aglomerado urbano do estado - abriga $73,7 \%$ dos casos. A raça parda teve maior frequência entre os doentes. Quanto à ocupação evidenciou-se que os desempregados (25\%) estavam em maior proporção, seguido das donas de casa $(19,7 \%)$ e trabalhadores autônomos (14,5\%). A maioria dos pacientes $(62.4 \%)$ tinha menos de sete anos de estudo.

Em relação à faixa etária dos pacientes em tratamento para TBMDR, houve maior predominância dos adultos jovens entre 20 e 39 anos (47,7\%) seguido daqueles entre 40 e 60 anos (43\%). No que tange ao tipo de resistência encontrado, a tabela 01 evidencia que 134 desses indivíduos possuíam resistência secundária $(88,2 \%)$, em comparação a apenas 18 com resistência primária $(11,8 \%)$ (Tabela 01).

Quanto ao consumo de drogas entre os indivíduos do estudo o uso do álcool foi o mais frequente $(20,4 \%)$, seguido das drogas ilícitas (16,5\%) e tabaco $(5,3 \%)$. 
Tabela 01 - Avaliação do tipo de resistência.

\begin{tabular}{lcc}
\hline Tipo de Resistência & $\mathbf{N}$ & $\%$ \\
\hline Primária & 18 & 11,8 \\
Secundária & 134 & 88,2 \\
\hline $\begin{array}{l}\text { Fonte: Coleta de dados } \\
\text { Frequência relativa. }\end{array}$
\end{tabular}

Quanto à distribuição percentual das reações adversas evidencia-se que as de maior frequência foram artralgia (31,6\%), epigastralgia (15,1\%) e neuropatia periférica $(5,3 \%)$. A ausência de comorbidades em nossa amostra foi mais frequente $(72,4 \%)$ (Tabela 02), sendo a comorbidade mais frequente o Diabetes Melitus (23\%) (Tabela 03).

Tabela 02 - Presença de comorbidade e reação adversa.

\begin{tabular}{lll}
\hline \multicolumn{1}{c}{ Variável } & $\mathbf{N}$ & $\%$ \\
Reação adversa & & 50 \\
Não & 76 & 50 \\
Sim & 76 & 72,4 \\
\hline Comorbidade & 110 & 27,6 \\
Não & 42 & \\
Sim & Fonte: Coleta de dados & -HUJBB. Belém, Pará, 2010-2015.N-Frequência absoluta; \% - \\
\hline Frequência relativa.
\end{tabular}

Em nossa amostra o desfecho clínico favorável, conforme caracterizado em nosso método, foi o mais frequente $(77 \%)$. É possível observar que nas variáveis sócio epidemiológicas somente a ocupação obteve significância estatística $(p=0,046)$. Podemos destacar que para todas as ocupações a maioria apresentou desfecho favorável, porém os desempregados tiveram o maior percentual de desfecho desfavorável $(39,5 \%$; $\mathrm{p}=0,046)$.

$\mathrm{Na}$ associação entre a presença de comorbidades e eventos adversos com o desfecho clínico, a maioria apresentou desfecho favorável $(88,1 \% ; \mathrm{p}=0,044)$. Entre aqueles que não apresentavam comorbidade associada, a maioria apresentou desfecho também favorável $(72,3 \% ; p=0,044)$, porém em menor proporção.

$\mathrm{Na}$ avaliação da associação entre o uso de drogas e o desfecho clínico foi possível avaliar que os usuários de drogas ilícitas e tabaco apresentaram maior frequência de desfecho desfavorável: drogas ilícitas (56,0\%; $\mathrm{p}<0,001)$; tabaco $(62,5 \% ; \mathrm{p}=0,006)$. Já para os usuários de álcool, a maioria apresentou desfecho favorável $(54,8 \%, p=0,001)$. 
Tabela 03 - Presença de comorbidade e reação adversa..

\begin{tabular}{|c|c|c|}
\hline Variável & $\mathbf{N}$ & $\%$ \\
\hline \multicolumn{3}{|l|}{ Convulsão } \\
\hline Não & 151 & 99,3 \\
\hline Sim & 1 & 50 \\
\hline \multicolumn{3}{|c|}{ Alteração dermatológica } \\
\hline Não & 152 & 100 \\
\hline Sim & 0 & 0 \\
\hline \multicolumn{3}{|l|}{ Epigastralgia } \\
\hline Não & 129 & 84,9 \\
\hline Sim & 23 & 15,1 \\
\hline \multicolumn{3}{|l|}{ Alteração auditiva } \\
\hline Não & 149 & 98 \\
\hline Sim & 3 & 2 \\
\hline \multicolumn{3}{|l|}{ Alteração mental } \\
\hline Não & 148 & 97,4 \\
\hline Sim & 4 & 2,6 \\
\hline \multicolumn{3}{|l|}{ Alteração visual } \\
\hline Não & 152 & 100 \\
\hline Sim & 0 & 0 \\
\hline \multicolumn{3}{|l|}{ Cefaléia } \\
\hline Não & 151 & 99,3 \\
\hline Sim & 1 & 0,7 \\
\hline \multicolumn{3}{|l|}{ Insônia } \\
\hline Não & 152 & 100 \\
\hline Sim & 0 & 0 \\
\hline \multicolumn{3}{|l|}{ Artralgia } \\
\hline Não & 104 & 68,4 \\
\hline Sim & 48 & 31,6 \\
\hline \multicolumn{3}{|c|}{ Neuropatia periférica } \\
\hline Não & 144 & 94,7 \\
\hline Sim & 8 & 5,3 \\
\hline \multicolumn{3}{|l|}{ Anafilaxia } \\
\hline Não & 150 & 98,7 \\
\hline Sim & 2 & 1,3 \\
\hline \multicolumn{3}{|l|}{ Vertigem/Nistagmo } \\
\hline Não & 149 & 98 \\
\hline Sim & 3 & 2 \\
\hline \multicolumn{3}{|l|}{ Náusea/Vômito } \\
\hline Não & 151 & 99,3 \\
\hline $\operatorname{Sim}$ & 1 & 0,7 \\
\hline \multicolumn{3}{|l|}{ Outros } \\
\hline Não & 144 & 94,7 \\
\hline Sim & 8 & 5,3 \\
\hline
\end{tabular}

Frequência relativa.

\section{DISCUSSÃO}

Em 2016, foram diagnosticados e registrados 66.796 casos novos e 12.809 casos de retratamento de tuberculose no Brasil (BRASIL, 2017). De acordo com o último relatório da OMS, o Brasil diagnosticou 63\% dos casos de tuberculose multirresistente estimados para 2015, valor superior ao ano anterior, que apresentava 40,0\% (WHO, 2016). Considerando-se apenas os casos novos notificados no SITE-TB e que iniciaram o tratamento para Tuberculose Resistente, 177 (23,5\%) eram de monorresistência, $330(43,9 \%)$ de resistência à rifampicina pelo TRM-TB, $49(6,5 \%)$ de polirresistência, $193(25,7 \%)$ de multirresistência e 3 (0,3\%) não tinham informação (BRASIL, 2017). 
O Pará é o estado da Região Norte com a segunda maior incidência de casos de TB, com média anual de 3.200 casos novos/ano, possui sete municípios considerados prioritários no país (BRASIL, 2011). No que tange a TBMDR o Pará encontra-se entre os 5 estados com maior incidência no país, junto com os estados do Rio de Janeiro, São Paulo, Bahia e Ceará. Na Região Norte, o Pará tem a maior incidência (306 casos), seguido do Amazonas (32 casos) e de Rondônia (25 casos) (SISTEMA TBMR, 2011).

Verificou-se que neste estudo os casos de TBMDR encontravam-se distribuídos em 31 municípios, sendo 27 no estado do Pará. Nos trabalhos semelhantes verificou-se que o maior número de casos se encontrava nas regiões metropolitanas e capitais. No presente trabalho a região metropolitana de Belém conta com $73,7 \%$ dos casos, resultados semelhantes foram encontrados por Mendes et al. (2014) que demostrou que no estado do Piauí $75,9 \%$ dos casos se concentram na capital Teresina. Assim como Ferreira et al. (2011) identificou que $74,2 \%$ dos pacientes com TBMDR no estado de São Paulo são procedentes da capital.

As regiões mais desenvolvidas dos estados contam com uma rede de saúde mais fortalecida, com mais recursos para a realização de exames e investigação de doenças. Esse fato possivelmente está relacionado ao fato dessas localidades apresentarem mais casos de TBMDR confirmados laboratorialmente somado ao fato 34 de que o acesso ao serviço de referência para acompanhamento e tratamento é mais acessível para a população. Porém, vale lembrar que aglomerados urbanos constituem fator de risco importante para transmissão da TBMDR primária (MENSCHHEIN et al., 2009).

O gênero masculino representa a maioria dos pacientes acometidos com TBMDR no presente casuístico, com $65,1 \%$, assim como demonstrado por Ferreira et al. (2011) uma predominância de $64,4 \%$ e por Vieira et al (2007), 70,2\%. Em relação à idade, a maioria dos pacientes deste estudo encontra-se com idade entre 20 e 39 anos (47,7\%), seguidos pela faixa etária dos 40 aos 60 anos (43\%). Melo et al (2003) também apresenta uma porcentagem importante de pacientes jovens, $73 \%$ deles estão entre 20 e 50 anos, constituindo uma predominância da doença na população economicamente ativa.

Em relação à etnia, este trabalho apresenta uma parcela significativa de pardos $(69,1 \%)$, seguido por brancos $(20,4 \%)$, negros $(9,2 \%)$ e um número menor de indígenas $(1,3 \%)$. Tais valores divergem do estudo realizado em Santa Catarina por Menschhein, Sandin, Sakae (2009) onde a raça branca é maioria (35,1\%), negros representam $16,7 \%$ e pardos $11,1 \%$, refletindo as variações étnicas no território nacional.

No presente estudo, nota-se a relevância da resistência secundária, que corresponde a $88,2 \%$ dos pacientes. Também foram encontrados valores elevados (97\%) nos estudos de Medeiros, Medeiros e Maciel (2008) e Siqueira et al. (2009) (84\%). Nos estados do Espírito Santo e Santa Catarina, os percentuais de resistência primária encontrados foram mais elevados, 19,3\% e 25,9\%, respectivamente. (VIEIRA et al., 2007; MENSCHHEIN et al., 2009). O baixo valor de resistência primária encontrado neste estudo $(11,8 \%)$ pode ser atribuído a não realização de rotina do teste de sensibilidade nesse serviço, ou seja, há uma deficiência na investigação de resistência no Pará se comparado a outros estados do país, porém encontrando-se dentro da média nacional (BARROSO et al., 2003).

No que tange a escolaridade, Juncks e D'Orsi (2008), em Santa Catarina, demonstraram que $64,4 \%$ tinham de 1 a 7 anos de estudo, valores próximos aos 35 encontrados em neste estudo (55,9\%). Situação semelhante ocorre em Teresina, onde Mendes et al. (2014) apresenta uma prevalência de 68,9\% para os indivíduos nessa mesma faixa de escolaridade. O baixo nível de escolaridade, provavelmente, é um fator que interfere na baixa assimilação das informações veiculadas pelo Ministério da saúde sobre a TB, seu diagnóstico e tratamento, bem como das orientações dadas pelos profissionais de saúde e na adesão ao tratamento, o que se relaciona diretamente às altas taxas de resistência secundária encontradas no país(MENSCHHEIN et al., 2009).

Neste trabalho, na variável ocupação, as donas de casa fazem parte de um percentual importante (19,7\%), assim como os desempregados (25\%). Ferreira et al. (2011) também apresentou em seus resultados uma alta frequência de desempregados (61,6\%). Juncks e D'Orsi (2008) alerta para o local do provável contágio, onde se destaca o contato intradomiciliar $(24,5 \%)$, sendo um importante fator de risco tanto para os desempregados quanto para as donas de casa. 
Juncks e D'Orsi (2008), em Santa Catarina, chama a atenção aos casos entre detentos $(10,2 \%)$ e à transmissão dentro dos presídios catarinenses (14,3\%). No estudo realizado por Almeida, Barbosa e Almeida (2013), onde foram considerados todos os casos novos de TBMR notificados ao Sistema de Informação de Agravos de Notificação (SINAN), ocorridos no Brasil entre 2008 e 2012, os autores demonstram que há uma parcela importante de pacientes presidiários correspondendo a $3,72 \%$ dos casos encontrados. No presente estudo os presidiários quantificam um número de $0,7 \%$ dos casos, demonstrando uma taxa inferior à média nacional.

Em relação ao consumo de drogas, as taxas encontradas neste estudo foram: álcool (20,4\%), drogas ilícitas (16,5\%) e tabaco (5,3\%). No estudo realizado no Rio de Janeiro, Ferreira et al. (2011) apresentou valores próximos sobre o alcoolismo $(22,9 \%)$, porém com percentual bem mais elevado de tabagistas $(28,2 \%)$. Já no trabalho de Juncks e D'Orsi (2008), 20,4\% dos indivíduos faziam uso de drogas ilícitas e 16,3\% eram etilistas. Vieira et al. (2007) encontrou no Espírito Santo percentuais bem mais elevados de alcoolismo $(59,5 \%)$ se comparado aos demais estudos.

A maioria dos indivíduos do presente estudo não possuía nenhuma comorbidade $(72,4 \%)$, dados semelhantes aos encontrados por Siqueira et al. (2007), no qual $76 \%$ da população estudada não apresentava comorbidades. O percentual de pacientes com comorbidade $(27,6 \%)$ foi inferior ao descrito para o Brasil, de 45,24\%, segundo dados coletados por Juncks e D'Orsi (2008) utilizando o Sistema de Informações de Agravos de Notificação (SINAN-NET) e sistema TBMR no período de 2001 a 2007.

Neste estudo o diabetes mellitus foi a comorbidade de maior frequência, presente em $23 \%$ dos pacientes. Ao comparar esse valor com outros estudos nacionais verificou-se que no estado do Ceará apenas $8 \%$ eram diabéticos, em Santa Catarina 3,7\% e em São Paulo 3\%. No trabalho de Karagöz et al. (2009), o DM foi a segunda comorbidade mais comum, presente em $12 \%$ dos pacientes. Esses dados evidenciam que no estado do Pará o número de diabéticos portadores de TBMR foi significativamente maior que em outros estados.

Historicamente, a incidência de TB em pacientes com diabetes é alta, sendo associado principalmente àqueles de difícil controle glicêmico. $O$ diabetes mellitus já é considerado um fator de risco para a tuberculose pulmonar e interfere negativamente tanto na apresentação da doença quanto na resposta ao tratamento (DOOLEY; CHAISSON,2009).

Bashar et al. (2001) em estudo sobre a incidência de TBMR em diabéticos relatou que o risco de os diabéticos desenvolverem tuberculose pulmonar é até cinco vezes maior que os não diabéticos, e demonstrou que $36 \%$ dos diabéticos desenvolveram TBMR comparado a apenas $10 \%$ do grupo controle dos não diabéticos. Dooley e Chaisson (2009) relata que o mecanismo científico pelo qual o diabetes mellitus levaria à aquisição preferencial de tuberculose multirresistente ainda não é claro.

Em relação ao tratamento, $50 \%$ dos pacientes do presente estudo foi acometido por algum tipo de reação adversa. Karagöz et al. (2009) obteve resultado semelhante, com 55,6\% apresentando reações adversas. Uma ou mais reações adversas foram observadas em 37.1\% da população estudada por Yang et al. (2017), na Coréia do Sul. As reações adversas mais frequentes foram: artralgia $(31,6 \%)$, epigastralgia $(15,1 \%)$ e neuropatia periférica (5,3\%). Quando comparados com o resultado de Yang et al. (2017), percentuais semelhantes de epigastralgia $(18,4 \%)$ são encontrados em sua população de estudo, porém com significativa menor frequência de artralgia (4,7\%).

No presente estudo 117 pacientes (77\%) obtiveram um desfecho favorável e 35 pacientes (23\%) um desfecho desfavorável, valores comparáveis aos encontrados em estudos realizados por Vieira et al. (2007) no Espírito Santo, no qual demonstrou que $71,7 \%$ e $28,3 \%$ da população do estudo teve obtenção de desfecho favorável e desfavorável, respectivamente. Viana (2010) relatou desfecho favorável em $71,6 \%$ dos pacientes atendidos em centro especializado no Maranhão.

De acordo com o Boletim epidemiológico da Secretaria de Vigilância em Saúde, divulgado neste ano de 2017, entre os casos novos de tuberculose multirresistente de $2014,66,5 \%$ curaram ou completaram o tratamento e $16,7 \% 38$ abandonaram o tratamento, resultados inferiores aos encontrados neste trabalho. 
O presente estudo mostra resultados significativos de desfecho desfavorável entre os usuários de drogas ilícitas, álcool e tabaco (56\%, 45,2\% e 62,5\%, respectivamente). Cassiano (2014), ao estudar a TB no estado do Ceará, apresenta uma taxa de abandono elevada entre os tabagistas $(58,5 \%)$, usuários de drogas $(56,1 \%)$ e alcoolistas $(68,3 \%)$.

Em relação às comorbidades, neste estudo foi encontrado menor índice de desfecho desfavorável naqueles que apresentavam comorbidade (11,9\%), do que naqueles sem comorbidade $(27,3 \%)$. Contrapondo-se a esse resultado um trabalho realizado no estado do Pará por Barros (2012) verificou-se que a maioria das pessoas com desfecho por cura não tinha comorbidades associadas.

No trabalho de Picon et al. (2011), realizado em Porto Alegre, estudou-se a população em retratamento para tuberculose, constatou-se que $45,4 \%$ dos pacientes fizeram uso irregular da medicação. A irregularidade foi maior entre os pacientes que apresentaram reações adversas gastrointestinais $(59,7 \%)$ do que entre aqueles que não as apresentaram (21,7\%). A taxa de cura foi significativamente menor nos pacientes que usaram irregularmente a medicação $(30,8 \%)$ e nos que apresentaram reações adversas $(53,7 \%)$. Dentre as reações adversas, apenas as gastrointestinais 39 mostraram-se relacionadas com menores taxas de cura, com valores de $51,5 \%$, enquanto que os pacientes que não apresentaram reações gastrointestinais esta taxa foi de $66,9 \%$.

Quando foi comparado o desfecho clínico com variáveis que poderiam interferir no mesmo, verificou-se que não houve significância estatística para as variáveis: gênero, tipo de resistência e reações adversas, embora os resultados mostrem que a maioria das pessoas com desfecho por cura era do gênero feminino, apresentava reação adversa e possuía resistência adquirida.

\section{CONCLUSÃO}

No perfil clínico-epidemiológico dos pacientes com TBMDR, em um serviço de referência no estado do Pará, houve predomínio do gênero masculino, adulto jovem, desempregados, baixa escolaridade e vivendo na região metropolitana de Belém, com o tipo de resistência predominante, a resistência secundária e verificou-se que etilismo, uso de drogas ilícitas e tabagismo foram hábitos frequentes entre os pacientes.

Metade dos pacientes apresentou algum tipo de reação adversa, sendo que as mais frequentes foram artralgia, epigastralgia e neuropatia periférica. Dentre as comorbidades associadas, o diabetes mellitus teve significativa frequência na população do estudo (23\%), bem superior a todos os outros trabalhos analisados.

A maioria dos pacientes teve desfecho clínico favorável e dentre as variáveis estudadas as únicas que tiveram impacto no desfecho clínico com significância estatística foram: ocupação, comorbidades, uso de drogas ilícitas, tabagismo e etilismo. Concluiu-se que a presença de rações adversas ao tratamento da TBMR não esteve relacionada com maior frequência de desfechos desfavoráveis nessa população, e que o tratamento realizado hoje no manejo da TBMR neste serviço é seguro e possui altas taxas de desfechos favoráveis.

\section{REFERÊNCIAS}

1. ALMEIDA MG, BARBOSA DRM, ALMEIDA DFS et al. Epidemiologia e distribuição espacial de casos notificados de tuberculose multirresistente (TBMR) no Brasil, 2008-2012,Rev Epidemiol Control Infect.2013; 3(4):117-122.

2. BASHAR M, ALCABES P., ROM WN et al. Increased Incidence of Multidrug-Resistant Tuberculosis in Diabetic Patients on the Bellevue Chest Service, 1987 to 1997. Chest 120: 1514-1519, 2001.

3. BARROS WA. Tendência histórica da tuberculose multirresistente, no programa de assistência do Hospital Universitário João de Barros Barreto. Dissertação (Mestrado) - Universidade Federal do Pará, Instituto de Ciências Biológicas, Programa de PósGraduação em Biologia de Agentes Infecciosos e Parasitários, Belém, 2012.

4. BARROSO EC et al. Papel da tuberculose domiciliar no surgimento da tuberculose multirresistente. J. bras. pneumol., São Paulo, v. 30, n. 1, p. 46- 52, Feb. 2004.

5. BARROSO EC et al. Fatores associados aos tratamentos inadequados em grupo de portadores de tuberculose multirresistente. Jornal de Pneumologia 29 (6): 350-357, 2003. 
6. BRASIL. Ministério da Saúde. Fundação Nacional de Saúde. Centro de Referência Professor Hélio Fraga; Sociedade Brasileira de Pneumologia e Tisiologia Controle da tuberculose: uma proposta de integração ensinoserviço. Rio de Janeiro: FUNASA/CRPHF/SBPT;2002. BRASIL. Ministério da Saúde. Secretaria de Vigilância em Saúde. Centro de Referência Professor Hélio Fraga. Projeto MSH. Tuberculose Multirresistente - Guia de Vigilância Epidemiológica - 1ª edição. Rio de Janeiro, 2007.

7. BRASIL. Ministério da Saúde. Secretária de Vigilância em Saúde. Departamento de Vigilância Epidemiológica. Programa Nacional de Controle da Tuberculose. Nota técnica sobre as mudanças no tratamento da tuberculose no Brasil para adultos e adolescentes. Brasília: Ministério da Saúde, 2009.

8. BRASIL, Ministério da Saúde. Nota técnica ํㅡ 5: Definição de critérios para a priorização de municípios no controle da tuberculose no Brasil. Municípios prioritários para o Controle de Tuberculose. Departamento de vigilância das doenças transmissíveis. Programa nacional de controle da tuberculose. Brasília: MS, 2011.

9. BRASIL. Ministério da Saúde. Secretaria de Vigilância em Saúde. Departamento de Vigilância Epidemiológica. Manual de recomendações para o controle da tuberculose no Brasil. Brasília: Ministério da Saúde, 2011. BRASIL. Ministério da Saúde. Secretaria de Vigilância em Saúde. Tratamento diretamente observado (TDO) da tuberculose na atenção básica: protocolo de enfermagem. Departamento de Vigilância Epidemiológica. Brasília, 2011.

10. BRASIL, Ministério da Saúde. Secretaria de Vigilância em Saúde. Programa Nacional de Controle da Tuberculose. Definição de critérios para a priorização de municípios no controle da tuberculose no Brasil, Brasília, 2011.

11. BRASIL. Ministério da Saúde. Secretaria de Vigilância em Saúde. Indicadores prioritários para o monitoramento do Plano Nacional pelo Fim da Tuberculose como Problema de Saúde Pública no Brasil. Boletim Epidemiológico, Brasília, 2017.

12. CAMINERO JA. A Tuberculosis Guide for Specialist Physicians. International Union Against Tuberculosis and Lung Disease (IUATLD). 68 boulevard Saint Michel, 75006, Paris - France. 2004.

13. DOOLEY KE, CHAISSON ER. Tuberculosis and diabetes mellitus: convergence of two epidemics. The Lancet Infectious Diseases 9 (12): 737- 746, 2009.

14. FERREIRA KR. Portadores de tuberculose multirresistente em um Centro de Referência: perfil sóciodemográfico e clínicoepidemiológico. Rev Esc Enferm USP 2011;

15. KARAGÖZ T,YAZICIOGLU MOÇIN O, PAZARLI P et al. The treatment results of patients with multidrug resistant tuberculosis and factors affecting treatment outcome. Tuberk Toraks. 2009;57(4):383-92.

16. LANGE C, MIGLIORI GB (Org). European Respiratory Monograph. Copyright ERS, n.58, 2012.

17. MEDEIROS JCM, MEDEIROS EM, MACIEL SS. V. Perfil epidemiológico dos clientes portadores de Tuberculose Multirresistente do Hospital Geral Otávio de Freitas: uma análise de janeiro de 2002 a janeiro de 2007. 2008. Monografia. (Aperfeiçoamento/Especialização em Saúde Pública) - Associação Caruaruense de Ensino Superior - ACES, Recife. 2008.

18. MELO FAF., AFIUNE JB, RIBEIRO LHG. Resistência primária do M. tuberculosis num serviço ambulatorial de referência em São Paulo: evolução por três décadas e comparação com outros estudos nacionais. Jornal de Pneumologia 22: 3-8, 1996.

19. MENSCHHEIN CL, SANDIN GR, SAKAE TM. Perfil epidemiológico dos casos de tuberculose multirresistente no estado de Santa Catarina entre 2004 e 2008. Revista Arquivos Catarinenses de Medicina (38) 4: 81-86, 2009.

20. MENDES MRR, COÊLHO DMM, SILVA JC et al. Situação sóciodemográfica da tuberculose multirresistente no estado do Piauí, 2001 - 2012. R. Interd. v. 7, n. 1, p. 8-16, jan. fev. mar. 2014.

21. MIGLIORI GB, LANGE C, GIRARDI E et al. Extensively drug-resistant tuberculosis is worse than multidrug-resistant tuberculosis: different methodology and settings, same results, Clin. Infect. Dis. 2008.

22. WORLD HEALTH ORGANIZATION. Guidelines For The Prevention Of Tuberculosis. In Health Care Facilities In Resource-Limited Settings. WHO/CDS/TB/99.269. 1999.

23. WHO/IUATLD. Global Project on Anti-Tuberculosis Drug Resistance Surveillance. Anti-tuberculosis drug resistance in the world: fourth global report. Geneva, 2008.

24. WORLD HEALTH ORGANIZATION. Guidelines for the Programmatic Management of Drug-Resistant Tuberculosis: Emergency Update 2008. Geneva: World Health Organization, Stop TB Department; 2008. 